\title{
On Removable Singularities of Complex Analytic Sets
}

\author{
A. B. SukHov
}

\begin{abstract}
This article considers boundary properties of complex analytic sets. The possibility of the continuation of complex analytic sets across a real submanifold in $\mathbf{C}^{n}$ whose Levi form is (in some sense) nondegenerate is shown.
\end{abstract}

0. Introduction. In this paper we study the well-known problem of the analytic continuation of complex analytic sets. There are two main directions in this problem: the local analytic continuation and the removal of singularities. Let $\Omega$ be a domain in $\mathbf{C}^{N}$ and $M$ is a closed subset in $\Omega$. Suppose that $A$ is a complex purely $q$-dimensional analytic subset in $\Omega \backslash M$ (as usual, we always suppose that $A$ is closed in $\Omega \backslash M)$. Then $M$ is called a removable singularity for $A$ if a closure $\bar{A}$ of $A$ (relatively $\Omega$ ) is an analytic subset in $\Omega$. We say that a purely $q$-dimensional analytic subset $A$ of a domain $D \subset \mathbf{C}^{N}$ analytically continues to the neighborhood $U$ of a point $p \in \bar{A} \cap \partial D$ if there exists a complex purely $q$-dimensional subset $\tilde{A} \subset U$ such that $(A \cap U) \subset \tilde{A}$.

One of the most famous results about removable singularities is the Remmert-Stein theorem [Re, St], which guarantees the removal of $M$ under the condition that $M$ be a complex analytic set and $\operatorname{dim} M<\operatorname{dim} A$. Essentially, this result means that if a (real) metrical dimension of $M$ is sufficiently small in comparison with a real dimension of $A$, then $M$ is removable for $A$ (see [Sh1]). The notion of complex manifold is a partial case of the notion of CRmanifold (Cauchy-Riemann manifold). Recall that a real submanifold $M$ in $\mathbf{C}^{N}$ is called a CR-manifold if the dimension of its holomorphic tangent space does not depend on the choice of a point of $M$ (it is called a CR-dimension of $M$ and denotes $\mathrm{CR} \operatorname{dim} M)$. In [Be], [Sh2], [Fu], [Ch1] a significant fact was discovered, namely, that a $\mathrm{CR}$-manifold $M$ whose real dimension is arbitrarily more than the real dimension of $A$ can be removable for $A$ if the CR-dimension of $M$ is sufficiently less than $\operatorname{dim} A$. In the present paper we use the Levi form of a $\mathrm{CR}$-manifold instead of a CR-dimension. By comparison to the CR-dimension, the Levi form is essentially a more delicate characteristic of the biholomorphic 
geometry of a CR-manifold. This allows us to prove that a $C R$-manifold $M$ whose $C R$-dimension is arbitrarily more than $\operatorname{dim} A$ can be removable for $A$ if $M$ has sufficiently non-degenerate Levi form. The first step in this direction was made in $[\mathrm{Pi}, \mathrm{Su}]$. In the present paper we develop ideas from that article.

This paper considers the case when $M$ is a real generic manifold of real codimension $m \geq 2$ in a domain $\Omega \subset \mathbf{C}^{N}$. The Levi form Levi ${ }_{p}^{M}$ of $M$ (at a point $p \in M)$ is a Hermitian form defined on the holomorphic tangent space $H_{p}(M)$ and having values in $N_{p}(M)=T_{p}(M) / H_{p}(M)$ (where $T_{p}(M)$ is a real tangent space). Using the Levi form, we define the biholomorphic invariants $\ell d_{p}^{k}(M), k=1, \ldots, m$ ("the Levi-dimension of order $k$ ") of a manifold $M$ as follows: $\ell d_{p}^{k}(M)=\max h d_{p}^{k}\left(F_{k}\right)$ where the maximum is taken over all (real) $k$ dimensional linear subspaces $F_{k}$ of $N_{p}(M)$ and $h d_{p}^{k}\left(F_{k}\right)$ is the largest dimension of complex linear subspaces of $H_{p}(M)$ whose images under the Levi form action lie in $F_{k}$. In partial cases, such invariants were introduced in [Pi], [Pi, Su] and in [Ch3] in the present form. It is obvious that $\ell d_{p}^{k}(M) \leq \ell d_{p}^{t}(M)$ when $k<t$. The main object of our investigations is the conjecture (stated in [Ch3]) that if $q-1>\ell d_{p}^{1}(M)$ for every $p \in \bar{A} \cap M$, then $M$ is removable for a purely $q^{-}$ dimensional analytic subset $A$. The analogous fact was established in [Ch1] with a CR-dimension of $M$ instead of $\ell d_{p}^{1}(M)$. But it is clear that the CR-dimension of $M$ at $p \in M$ coincides with $\ell d_{p}^{m}(M)$. A stronger result was obtained in [Pi, $\mathrm{Su}$ ] where $\ell d_{p}^{m-1}(M)$ is used instead of the CR $\operatorname{dim} M$. So the conjecture seems quite natural.

In the present paper this conjecture is proved under an additional assumption on the Levi form of $M$, which will be taken to be $k$-pseudoconcave with $k>\mathrm{CR} \operatorname{dim} M-q+1$ (Theorem 3.1). The condition of $k$-pseudoconcavity means that the origin belongs to the convex hull of the set of those vectors of $\mathbf{R}^{m}$ in whose directions the Levi form of $M$ has no less than $k$ positive eigenvalues on $H_{p}(M)$ (Definitions 2.2 and 2.4). Using the Levi form we also introduce some local biholomorphic invariant $\operatorname{def}_{p}(M)$ ("the Levi-deffect") of $M$ with the property $\ell d_{p}^{1}(M) \leq \operatorname{def}_{p}(M) \leq \ell d_{p}^{m-1}(M)$. This invariant characterizes the nondegeneracy of the Levi form. We show the removal of $M$ under the condition $\operatorname{dim} A-1>\operatorname{def}_{p}(M)$ for every $p \in \bar{A} \cap M$ (Theorem 3.8). Note that our results essentially strengthen known results [Be], [Sh2], [Fu], [Ch1], [Pi, Su] (Remark $3.10)$.

This article is organized as follows. In Section 1 we introduce local biholomorphic invariants of CR-manifolds. In Section 2 we study the problem of local analytic continuation. In Section 3 we apply results of Section 2 and prove the main theorems of this article about removable singularities.

I am thankful to E. M. Chirka who attracted my attention to this problem and to S. I. Pinchuk for the interest to this work. I am also obliged to Y. V. Khurumov for useful discussions of obtained results. 
1. The Levi form and local biholomorphic invariants. Let $\Omega$ be a domain in $\mathbf{C}^{N}$ and $M$ a generic $C^{2}$-manifold of codimension $m \geq 2$ in $\Omega$. Then $M$ can be represented in the form

$$
M=\left\{z \in \Omega: \rho_{1}(z)=\cdots=\rho_{m}(z)=0\right\},
$$

where $\rho_{j} \in C^{2}(\Omega)$ and $\partial \rho_{1} \wedge \cdots \wedge \partial \rho_{m} \neq 0$ in $\Omega$. Let $T_{p}(M)$ denote the real tangent space of $M$ at a point $p \in M$ and let $H_{p}(M)=T_{p}(M) \cap J\left(T_{p}(M)\right)$ (where $J$ is the operator of the complex structure in $\mathbf{C}^{N}$ ) be the holomorphic tangent space. We denote the complex dimension of $H_{p}(M)$ as $\mathrm{CR} \operatorname{dim} M$. For generic manifolds it does not depend on $p \in M$ and is equal to $(N-m)$. The functions $\rho_{j}$ from (1.1) are called the defining functions of $M$. Also, let $\hat{\rho}(z)$ be a vector-valued function of the form $\hat{\rho}(z)=\left(\rho_{1}(z), \ldots, \rho_{m}(z)\right.$ ) (with values in $\mathbf{R}^{m}$.

Set $N_{p}(M)=T_{p}(M) / H_{p}(M)$. Then $N_{p}(M)$ is a real $m$-dimensional linear space since $M$ is generic. The Levi form can be defined as a map Levi ${ }_{p}^{m}$ : $H_{p}(M) \rightarrow N_{p}(M)$ of the form $\operatorname{Levi}_{p}^{M}(z)=\pi\left(\left(\frac{i}{2}\right)[V, \bar{V}](p)\right)$ where $\pi: T_{p}(M) \rightarrow$ $N_{p}(M)$ is the quotient map, $V$ is a vector field of the type $(1,0)$ having values in the holomorphic tangent bundle $H(M)\left(\cong H^{1,0}(M)\right)$ and $V(p)=z$. Certainly this definition does not depend on the choice of $V$ (e.g., see details in [Bo, Po], [Ch3]).

Now we are ready to define biholomorphic invariants of $M$. Let $F_{k}$ be a real $k$-dimensional subspace in $N_{p}(M)$. Let also $h d_{p}^{k}\left(F_{k}\right)$ be the largest complex dimension of a $\mathbf{C}$-linear subspace that contains a set of the following form:

$$
\left(\operatorname{Levi}_{p}^{M}\right)^{-1}\left(F_{k}\right)=\left\{z \in H_{p}(M): \operatorname{Levi}_{p}^{M}(z) \in F_{k}\right\} .
$$

Set

$$
\ell d_{p}^{k}(M)=\max _{F_{k}} h d_{p}^{k}\left(F_{k}\right)
$$

where the maximum is taken over all real $k$-dimensional linear subspaces of $N_{p}(M)$. Evidently, natural numbers $\ell d_{p}^{k}(M), k=1, \ldots, m$ are (local) biholomorphic invariants of $M$. If CR $\operatorname{dim} M=0$ ( $M$ is totally real), then $\ell d_{p}^{k}(M)=0$ for any $k$. It is also easy to see that in the non-trivial case when CR $\operatorname{dim} M>0$, we have $\ell d_{p}^{m}(M)=\mathrm{CR} \operatorname{dim} M$ and $1 \leq \ell d_{p}^{1}(M) \leq \cdots \leq \ell d_{p}^{m-1}(M) \leq \ell d_{p}^{m}(M)=$ $N-m$. We call $\ell d_{p}^{k}(M)$ the Levi-dimension of $M$ of order $k$ at $p$. It follows from above that the Levi-dimension is in some sense the generalization of a CR-dimension.

For $\alpha \in N_{p}(M)$ we set

$$
d_{p}(\alpha)=\min _{\Gamma_{\alpha}} h d_{p}^{m-1}\left(\Gamma_{\alpha}\right)
$$


where $\Gamma_{\alpha} \subset N_{p}(M)$ is an arbitrary real linear hypersurface with $\alpha \in \Gamma_{\alpha}$ and $0 \in \Gamma_{\alpha}$. Set also

$$
\operatorname{def}_{p}(M)=\max _{\alpha \in N_{p}(M)} d_{p}(\alpha)
$$

It is clear that $\operatorname{def}_{p}(M)$ also is a (local) biholomorphic invariant of $M$ and we have $\ell d_{p}^{1}(M) \leq \operatorname{def}_{p}(M) \leq \ell d_{p}^{m-1}(M)$. We call $\operatorname{def}_{p}(M)$ the Levi-defect of $M$ at a point $p$ (see Remark 1.11 below).

Let $L_{p}\left(\rho_{j}, \omega\right)$ denote a value of the Levi form (complex Hessian) of a defining function $\rho_{j}$ from (1.1) at a point $p \in M$ on a vector $\omega \in H_{p}(M)$. Then

$$
L_{p}\left(\rho_{j}, \omega\right)=\sum_{\nu, \mu=1}^{N} \frac{\partial^{2} \rho_{j}}{\partial z_{\nu} \partial \bar{z}_{\mu}}(p) \omega_{\nu} \bar{\omega}_{\mu} .
$$

It is easy to find the connection between the Levi form of $M$ and Levi forms of its defining functions. Let us consider a point $p \in M$. It is a well-known fact $[\mathrm{Bo}, \mathrm{Po}]$ that there is a neighborhood $U \ni p$ such that after some biholomorphic change of coordinates which transforms $p$ to 0 and $U$ to the neighborhood $U^{\prime} \ni 0$, we have in new coordinates (setting $w=u+i v$ ):

$$
\begin{aligned}
& M \cap U^{\prime}=\left\{(z, w) \in U^{\prime} \subset \mathbf{C}_{z}^{n} \times \mathbf{C}_{w}^{m}=\mathbf{C}^{N}:\right. \\
&\left.\rho_{j}=u_{j}+\varphi_{j}(z, v)=0, j=1, \ldots, m\right\},
\end{aligned}
$$

where for $\omega=(z, 0)$ we also have

$$
\varphi_{j}(z, v)=L_{0}\left(\rho_{j}, \omega\right)+o\left(|z|^{2}+|v|^{2}\right) .
$$

Then $H_{0}(M) \cong \mathbf{C}_{z}^{n}$ and $T_{0}(M) / H_{0}(M) \cong \mathbf{R}_{v}^{m}$ since $T_{o}(M)=\mathbf{C}_{z}^{n} \oplus \mathbf{R}_{v}^{m}$. Choosing the natural basis in $\mathbf{R}_{v}^{m}$, we obtain after standard calculations (see e. g., [Bo, Po], [Ch3]) that the Levi form can be identified (up to the scalar

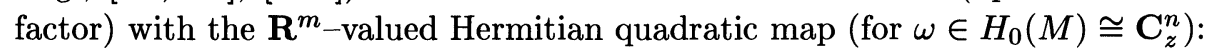

$$
\operatorname{Levi}_{0}^{M}(\omega) \cong\left(L_{0}\left(\rho_{1}, \omega\right), \ldots, \mathrm{L}_{0}\left(\rho_{m}, \omega\right)\right) .
$$

Such identification depends on the choice of basis in $\mathbf{R}^{m} \cong N_{0}(M)$ and therefore in reality it is necessary to consider the map (1.5) up to the multiplication on non-degenerate real $(m \times m)$-matrices. This makes quite natural the next interpretation of the Levi form.

Let $S^{m-1}$ be the unit sphere in $\mathbf{R}^{m}$. Let also $\langle\alpha, \beta\rangle=\sum_{j=1}^{m} \alpha_{j} \beta_{j}$ be the scalar product in $\mathbf{R}^{m}$. For $M$ of the form (1.1), let us consider the following family of Hermitian forms:

$$
L_{p}(\langle\alpha, \hat{\rho}\rangle, \omega)=\sum_{j=1}^{m} \alpha_{j} L_{p}\left(\rho_{j}, \omega\right),
$$


where $\alpha=\left(\alpha_{1}, \ldots, \alpha_{m}\right) \in S^{m-1}$ and $\omega \in H_{p}(M)$.

Well-known properties of the complex Hessian show that this family does not depend on the choice of defining functions (1.1) and it is invariant under the biholomorphic changes of coordinates. So the family (1.6) essentially can be identified with the Levi form. To calculate the invariants introduced above, it is convenient to express them in terms of the family (1.6).

For linearly independent $\beta^{1}, \ldots, \beta^{t} \in S^{m-1}$, let $s_{p}^{t}\left(\beta^{1}, \ldots, \beta^{t}\right)$ denote the largest complex dimension of $\mathbf{C}$-linear subspaces of the following set:

$$
\left\{z \in H_{p}(M): L_{p}\left(\left\langle\beta^{j}, \hat{\rho}\right\rangle, z\right)=0, j=1, \ldots, t\right\} .
$$

Proposition 1.8. Let $p \in M$. Then:

$$
\ell d_{p}^{k}(M)=\max s_{p}^{m-k}\left(\beta^{1}, \ldots, \beta^{m-k}\right), \quad k=1, \ldots, m-1,
$$

where the maximum is taken over all linearly independent systems of vectors $\beta^{1}, \ldots, \beta^{m-k} \in S^{m-1}$.

$$
\operatorname{def}_{p}(M)=\max _{\alpha \in S^{m-1}} \tilde{d}_{p}(\alpha)
$$

where $\tilde{d}_{p}(\alpha)=\min s_{p}^{1}(\beta)$ and the minimum is taken over all $\beta \in S^{m-1}$ such that $\langle\alpha, \beta\rangle=0$.

Proof. Without any loss of generality, we may assume that $M$ is in the form (1.3), (1.4) with $p=0$. Let $F_{k}$ be a subspace of $N_{0}(M) \cong \mathbf{R}^{m}$ of dimension $k$ and let $\beta^{1}, \ldots, \beta^{m-k}$ be a basis of its orthogonal complement in $\mathbf{R}^{m}$. Then (1.5), (1.6) implies that sets (1.2) and (1.7) coincide and (i) is established. Particularly if $\Gamma_{\alpha}$ is a hyperspace in $\mathbf{R}^{m}$ which contains $\alpha$ and $\langle\alpha, \beta\rangle=0$, then $s_{0}^{1}(\beta)=h d_{0}^{m-1}\left(\Gamma_{\alpha}\right)$ and $\tilde{d}_{0}(\alpha)=d_{0}(\alpha)$. It implies (ii).

Evidently we have $\ell d_{p}^{1}(M)=\cdots=\ell d_{p}^{m-1}(M)=\operatorname{def}_{p}(M)$ when $m=2$. Certainly it is not true in the general case.

Example 1.9. Let

$$
M=\left\{(z, w) \in \mathbf{C}^{n} \times \mathbf{C}^{n}: \operatorname{Re} w_{j}+\left|z_{j}\right|^{2}=0, j=1, \ldots, n\right\} .
$$

Then $\operatorname{def}_{0}(M)=1$ and $\ell d_{0}^{k}=k$ for $k=1, \ldots, n$.

Example 1.10. Set

$$
\begin{aligned}
M=\left\{(z, w) \in \mathbf{C}^{3} \times \mathbf{C}^{4}:\right. & \operatorname{Re} w_{1}+\left|z_{1}\right|^{2}-\left|z_{2}\right|^{2}=0, \\
& \operatorname{Re} w_{2}+\operatorname{Re} z_{1} \bar{z}_{2}=0, \\
& \operatorname{Re} w_{3}+\operatorname{Im} z_{1} \bar{z}_{2}=0, \\
& \left.\operatorname{Re} w_{4}+\left|z_{3}\right|^{2}=0\right\} .
\end{aligned}
$$

Then $\operatorname{def}_{0}(M)=2$ and $\ell d_{0}^{1}(M)=1$. 
Remark 1.11. The geometrical sense of introduced invariants is quite simple: they characterize the nondegeneracy of the Levi form of $M$. For example, if functions (1.1) are pluriharmonic ( $M$ is Levi-flat), then $\operatorname{def}_{p}(M)$ assumes its maximal admissible value and is equal to $\mathrm{CR} \operatorname{dim} M$. From the other side if $\operatorname{def}_{p}(M)$ (or $\ell d_{p}^{1}(M)$ ) is small in comparison with CR $\operatorname{dim} M$, then $M$ has essentially non-trivial Levi form.

2. Local analytic continuation. Recall the theorem of W. Rothstein on the continuation of analytic sets across pseudoconcave hypersurfaces [Ch2], [Rot]: Let $A$ be a (closed) purely q-dimensional subset in a domain of the form $\left\{z \in \mathbf{C}^{N}: \psi(z)>0\right\}$ where $\psi(p)=0$ and $p$ is a limit point of $A$. Suppose that the Levi form of $\psi$ at $p$ has no less than $(N-q+1)$ positive eigenvalues on the holomorphic tangent space of hypersurface $\left\{z \in \mathbf{C}^{N}: \psi(z)=0\right\}$ at $p$. Then $A$ analytically continuates to the neighborhood of $p$. In this section we shall use this result for the continuation across manifolds of an arbitrary codimension.

Let $\Omega$ be a domain in $\mathbf{C}^{N}$ and let $M \subset \Omega$ be a generic $C^{2}$-manifold of the form (1.1) in some neighborhood of $p \in M$.

Definition 2.1. We call $M k$-convex at $p \in M$ in the direction of $\sigma \in \mathbf{R}^{m}$ if the Hermitian quadratic form $L_{p}(\langle\sigma, \hat{\rho}\rangle, \omega), \omega \in H_{p}(M)$ has no less than $k$ positive eigenvalues.

Recall that a set $V \subset \mathbf{R}^{m}$ is called a cone if for every $v \in V$ and every $\lambda>0$ we have $\lambda v \in V$. Let also

$$
V^{*}=\left\{\alpha \in \mathbf{R}^{m}:\langle\alpha, \beta\rangle \geq 0 \text { for all } \beta \in V\right\}
$$

be the conjugate cone. Note that $V^{*}$ always is a closed convex cone and $0 \in V^{*}$.

Let $M$ be a manifold of the form (1.1) and let $V$ be an open cone in $\mathbf{R}^{m}$ with the non-zero conjugate cone $V^{*}$. Also, let $p$ be an arbitrary point of $M$. A domain $M(p, r, V)$ is called a standard domain if

$$
M(p, r, V)=\left\{z \in \mathbf{C}^{N}:|z-p|<r, \hat{\rho}(z) \in V\right\}
$$

Below we also use the notation $B^{N}(p, r)=\left\{z \in \mathbf{C}^{N}:|z-p|<r\right\}$. Note also that $0 \in \bar{V} \backslash V$ since $V^{*} \neq\{0\}$, and consequently $M \cap B^{N}(p, r) \subset \overline{M(p, r, V)} \backslash$ $M(p, r, V)$ for sufficiently small $r>0$.

Definition 2.4. Let $M$ be a generic $C^{2}$-manifold of the form (1.1) in some neighborhood of $p \in M$ and let $M(p, r, V)$ be a standard domain of the form (2.3). We call $M(p, r, V) k$-pseudoconcave at $p$ if $M$ is $k$-convex at $p$ in the direction of some $\sigma \in V^{*} \backslash\{0\}$. 
Proposition 2.5. Let $M$ be a generic $C^{2}$-manifold of the form (1.1) and let $A$ be a complex purely q-dimensional analytic subset in $B^{N}(p, r) \backslash M$ (for some $r>0)$. Suppose that for some open cone $V \subset \mathbf{R}^{m}$ with $V^{*} \neq\{0\}$ the standard domain $M(p, r, V)$ is $(N-m-q+2)$-pseudoconcave at $p \in M$ and $A \cap M\left(p, r^{\prime}, V\right)$ is non-empty for every $r^{\prime}>0$. Then the set $A \cap M(p, r, V)$ analytically continues to the neighborhood of $p$.

Proof. Let $\sigma \in V^{*} \backslash\{0\}$ be the vector for which the Levi form of the function $\langle\sigma, \hat{\rho}\rangle=\sum_{j=1}^{m} \sigma_{j} \rho_{j}$ has no less than $(N-m-q+2)$ positive eigenvalues on $H_{p}(M)$. Then we can choose some constant $C>0$ such that the Levi form of the function $\psi=\langle\sigma, \hat{\rho}\rangle+\mathbf{C} \sum_{j=1}^{m} \rho_{j}^{2}$ has no less than $(N-q+1)$ positive eigenvalues on the holomorphic tangent space of the hypersurface $\left\{z \in \mathbf{C}^{N}: \psi(z)=0\right\}$ at the point $p$. Let us consider the domain $D(r)=\left\{z \in B^{N}(p, r): \psi(z)>0\right\}$. It is clear that $A \cap M\left(p, r^{\prime}, V\right) \subset A \cap D\left(r^{\prime}\right)$ for sufficiently small $r^{\prime}>0$ and by the theorem of W. Rothstein there are $r^{\prime \prime}>0$ and a complex $q$-dimensional analytic set $\tilde{A} \subset B^{N}\left(p, r^{\prime \prime}\right)$ such that $A \cap D\left(r^{\prime \prime}\right) \subset \tilde{A}$. Then $\tilde{A}$ is a local analytic continuation of $A \cap M(p, r, V)$.

Let $M$ be a manifold of the form (1.1) in some neighborhood of $p \in M$. Also, let $Q_{p}(k)$ denote the set of all $\alpha \in S^{m-1}$ such that $M$ is $k$-convex in the direction of every $\alpha \in Q_{p}(k)$. For an arbitrary set $X \subset \mathbf{R}^{m}$ we consider its convex conic hull $C o(X)$. Recall that $C o(X)$ is the set of all finite linear combinations of vectors of $X$ with non-negative coefficients and in every combination coefficients are not equal to zero simultaneously.

Definition 2.6. A generic $C^{2}$-manifold $M$ of the form (1.1) is called $k-$ pseudoconcave at $p \in M$ if

$$
0 \in C o\left(Q_{p}(k)\right)
$$

Since the family (1.6) of Levi forms does not depend on the choice of the system of defining functions of $M$, Definition 2.6 also does not depend on the choice of defining functions.

Propostion 2.8. A generic $C^{2}$-manifold $M$ of the form (1.1) is $k$-pseudoconcave at $p \in M$ if and only if there exists $r>0$ and a finite collection of open cones $V_{j} \subset \mathbf{R}^{m}, j=1, \ldots, t$ such that corresponding standard domains $M\left(p, r, V_{j}\right)$ are $k$-pseudoconcave at $p$ and

$$
B^{N}(p, r) \backslash M=\bigcup_{j=1}^{t} M\left(p, r, V_{j}\right) .
$$

The proof uses the statement below. 
Lemma 2.10. A manifold $M$ of the form (1.1) is $k$-pseudoconcave at $p$ if and only if there exists a finite collection of vectors $\sigma_{j} \in Q_{p}(k), j=1, \ldots, t$ such that

$$
\mathbf{R}^{m}=\bigcup_{j=1}^{t}\left\{\alpha \in \mathbf{R}^{m}:\left\langle\sigma_{j}, \alpha\right\rangle \geq 0\right\}
$$

Proof. By the duality (2.11) is equivalent to that fact that the set

$$
\bigcap_{j=1}^{t}\left\{\alpha \in \mathbf{R}^{m}:\left\langle\sigma_{j}, \alpha\right\rangle<0\right\}
$$

is empty. But the system of linear inequalities (2.12) is non-compatible if and only if there exist real numbers $\lambda_{j} \geq 0$ that are not simultaneously equal to zero and such that $\sum_{j=1}^{t} \lambda_{j} \sigma_{j}=0$ ([R], Section 22, Theorem 22.2). This is equivalent to $(2.7)$.

Proof of Proposition 2.8. It is sufficient to prove that (2.9) and (2.11) are equivalent. It is clear that the cones $V_{j}$ satisfy (2.9) (with sufficiently small $r>0$ ) if and only if $\bigcup_{j=1}^{t} V_{j}=\mathbf{R}^{m} \backslash\{0\}$. But the pseudoconcavity of $M\left(p, r, V_{j}\right)$ implies that, for some $\sigma_{j} \in Q_{p}(k)$, we have $V_{j} \subset\left\{\alpha \in \mathbf{R}^{m}:\left\langle\sigma_{j}, \alpha\right\rangle \geq 0\right\}$. Therefore (2.11) is a consequence of (2.9). On the other hand, by the theorem on the continuous dependence of eigenvalues from a parameter ([Ho,Jo], Appendix D), the set $Q_{p}(k)$ is open in the standard topology of the sphere $S^{m-1}$. Therefore, from (2.11), for every $\sigma_{j}$ there is the neighborhood $\Delta_{j} \subset Q_{p}(k)$ such that

$$
\left\{\alpha \in \mathbf{R}^{m}:\left\langle\sigma_{j}, \alpha\right\rangle \geq 0\right\} \subset \bigcup_{\sigma \in \Delta_{j}}\left\{\alpha \in \mathbf{R}^{m}:\langle\sigma, \alpha\rangle>0\right\} .
$$

Then, from (2.11) it follows that

$$
S^{m-1} \subset \bigcup_{\sigma \in Q_{p}(k)}\left\{\alpha \in \mathbf{R}^{m}:\langle\sigma, \alpha\rangle>0\right\}
$$

and by the compactness of the sphere there exists a finite collection of vectors $\sigma_{j}^{\prime} \in Q_{p}(k), j=1, \ldots, t^{\prime}$ such that

$$
S^{m-1} \subset \bigcup_{j=1}^{t^{\prime}}\left\{\alpha \in \mathbf{R}^{m}:\left\langle\sigma_{j}^{\prime}, \alpha\right\rangle>0\right\}
$$

Then, the open cones $V_{j}=\left\{\alpha \in \mathbf{R}^{m}:\left\langle\sigma_{j}^{\prime}, \alpha\right\rangle>0\right\}$ satisfy (2.9) for some $r>0$ and $M\left(p, r, V_{j}\right)$ are $k$-pseudoconcave. The equivalence of (2.9) and (2.11) is proved. Now Proposition 2.8 follows from Lemma 2.10 .

As a consequence we obtain our first main result. 
Theorem 2.13. Let $\Omega$ be a domain in $\mathbf{C}^{N}$ and let $M$ be a generic $C^{2}$-manifold of codimension $m \geq 2$ in $\Omega$. Suppose that $A$ is a complex purely $q-$ dimensional analytic subset in $\Omega \backslash M$ with $p \in \bar{A} \cap M$ and $M$ is $(N-m-q+2)$ pseudoconcave at $p$. Then $A$ analytically continuates to some neighborhood of $p$.

Proof. From (2.9) it follows that the intersection of $A$ with at least one of domains $M\left(p, r, V_{j}\right)$ is non-empty for every $r>0$. By Proposition 2.5, there is, for such $j, r^{\prime}>0$ and the purely $q$-dimensional analytic subset $\tilde{A}_{j}$ in the ball $B^{N}\left(p, r^{\prime}\right)$ such that $A \cap M\left(p, r^{\prime}, V_{j}\right) \subset \tilde{A}_{j}$. Consider the analytic purely $q^{-}$ dimensional set $\tilde{A}=\bigcup_{j} \tilde{A}_{j}$, where the union is taken over all $j=1, \ldots, t$ such that $A \cap M\left(p, r, V_{j}\right)$ is not empty for every $r>0$. Then $\tilde{A}$ is the local analytic continuation of $A$ to some neighborhood of $p$.

Now we shall find the connection between pseudoconcavity and the invariants of Section 1.

Theorem 2.14. Let $M$ be a generic $C^{2}{ }_{-}$manifold of codimension $m \geq 2$ in a domain $\Omega \subset \mathbf{C}^{N}$. Then $M$ is $\left(N-m-\operatorname{def}_{p}(M)\right)$-pseudoconcave for every $p \in M$.

Proof. Set $k=N-m-\operatorname{def}_{p}(M)$.

Lemma 2.15. For every $\alpha \in S^{m-1}$ there is $\beta \in Q_{p}(k)$ orthogonal to $\alpha$.

Proof of the Lemma. We use the notation of Proposition 1.8. From (ii) of Proposition 1.8 it follows that for every $\alpha \in S^{m-1}$ there is $\beta \in S^{m-1}$ orthogonal to $\alpha$ such that

$$
s_{p}^{1}(\beta)=d_{p}(\alpha) \leq \operatorname{def}_{p}(M)
$$

But it is clear that

$$
s_{p}^{1}(\beta)=\operatorname{ze}(\beta)+\min \{p o(\beta), \operatorname{ne}(\beta)\}
$$

where $\operatorname{po}(\beta), \operatorname{ne}(\beta), \operatorname{ze}(\beta)$ is the number of positive, negative and zero eigenvalues of $L_{p}(\langle\beta, \hat{\rho}\rangle, z), z \in H_{p}(M)$, respectively. Since ze $(\beta)=N-m-\operatorname{po}(\beta)-\operatorname{ne}(\beta)$, we obtain from $(2.16),(2.17)$ that $\max \{\operatorname{po}(\beta), \operatorname{ne}(\beta)\} \geq k$.

But po $(-\beta)=\operatorname{ne}(\beta)$ and consequently $\beta$ or $(-\beta)$ belongs to $Q_{p}(k)$. 
Let us consider an arbitrary $\alpha \in S^{m-1}$ and the corresponding $\beta$ from the Lemma 2.15. Since $Q_{p}(k)$ is open in the topology of the sphere $S^{m-1}$ in every neighborhood of $\beta$ there is a point $\beta^{\prime} \in Q_{p}(k)$ such that $\alpha \in V_{\alpha}=$ $\left\{\tau \in \mathbf{R}^{m}:\left\langle\beta^{\prime}, \tau\right\rangle>0\right\}$. Therefore, $S^{m-1} \subset \bigcup_{\alpha \in S^{m-1}} V_{\alpha}$ and choosing some finite subcovering, we obtain that there is a finite collection of vectors $\sigma_{j} \in Q_{p}(k)$, $j=1, \ldots, t$ such that

$$
S^{m-1}=\bigcup_{j=1}^{t}\left\{\alpha \in \mathbf{R}^{m-1}:\left\langle\sigma_{j}, \alpha\right\rangle>0\right\}
$$

This means that $\sigma_{j}$ satisfy (2.11). Therefore by Lemma $2.10 M$ is $k$-pseudoconcave at $p$.

The next result is the obvious consequence of Theorem 2.13 and 2.14.

Theorem 2.18. Let $M$ be a generic $C^{2}$-manifold of codimension $m \geq 2$ in a domain $\Omega \subset \mathbf{C}^{N}$ and let $A$ be a complex purely $q$-dimensional analytic subset in $\Omega \backslash M$. Suppose that for some point $p \in \bar{A} \cap M$ we have

$$
q-1>\operatorname{def}_{p}(M)
$$

Then A analytically continuates to some neighborhood of $p$.

Remark 2.20. Let us consider $M$ from Example 1.10. Then

$$
Q_{0}(2) \subset\left\{\left(\alpha_{1}, \alpha_{2}, \alpha_{3}, \alpha_{4}\right) \in \mathbf{R}^{4}: \alpha_{4}>0\right\}
$$

and therefore $M$ is not 2-pseudoconcave at 0 . This example shows that in general $\operatorname{def}_{p}(M)$ cannot be replaced by $\ell d_{p}^{1}(M)$ in Theorem 2.14 .

3. Removable singularities. Our first main result is the following.

Theorem 3.1. Let $M$ be a generic $C^{2}-$ manifold of codimension $m \geq 2$ in a domain $\Omega \subset \mathbf{C}^{N}$ and let $A$ be a complex purely $q$-dimensional analytic subset in $\Omega \backslash M$. Suppose that for every point $p \in \bar{A} \cap M$ we have

$$
q-1>\ell d_{p}^{1}(M)
$$

and $M$ is $(N-m-q+2)-p s e u d o c o n c a v e$ at $p$. Then the closure $\bar{A}$ of $A$ is an analytic subset in $\Omega$.

Note that the condition of pseudoconcavity without (3.2) is not sufficient for the removal of singularities. 
Example 3.3. Set

$$
\begin{aligned}
M=\left\{(z, w) \in \mathbf{C}^{3} \times \mathbf{C}^{2}:\right. & \operatorname{Re} w_{1}+\left|z_{1}\right|^{2}-\left|z_{2}\right|^{2}=0 \\
& \left.\operatorname{Re} w_{2}-\left|z_{2}\right|^{2}+\left|z_{3}\right|^{2}=0\right\} .
\end{aligned}
$$

Then $M$ is the 2-pseudoconcave manifold at every point $p \in M$. But for the 3-dimensional $A$ of the form $A=\left\{(z, w): z_{2}=z_{3}, w_{2}=0\right\}$ the set $A \backslash M$ is not connected. Therefore every connected component of $A \backslash M$ is a complex manifold whose boundary lies on $M$ and $M$ is not removable for analytic set which coincides with one (any) of such components. Note that here $\ell d_{p}^{1}(M)=2$ and (3.2) is not satisfied.

The following two statements are necessary for the proof of Theorem 3.1.

Lemma 3.4. Let $M$ be a generic $C^{2}$-manifold of the form (1.1) in $\mathbf{C}^{N}$ of codimension $m \geq 2$ and let $A$ be a complex $q$-dimensional manifold in some neighborhood of a point $p \in \bar{A} \cap M$. Suppose that (3.2) is satisfied at $p$ and the tangent space $T_{p} A$ does not lie in the $H_{p}(M)$. Then for every sufficiently small $r>0$ the set $(A \backslash M) \cap B^{N}(p, r)$ is linearly connected.

Proof. By the condition of the lemma we may assume that $d \rho(p) \mid T_{p} A \neq 0$. Also, without the loss of generality we can suppose that $p=0$ and coordinates (1.3), (1.4) are chosen. For some $r>0$ we have $A \cap M \cap B^{N}(0, r)=A \cap \Gamma_{m} \cap$ $B^{N}(0, r)$ where $\Gamma_{m}=\left\{z \in \mathbf{C}^{N}: \rho_{m}(z)=0\right\}$. Then by the division lemma ([Sha], Section 31, p. 211) there are $r^{\prime}<r$ and functions $h_{j} \in C^{1}\left(A \cap B^{N}\left(0, r^{\prime}\right)\right)$ such that

$$
\rho_{j}\left|\left(A \cap B^{N}\left(0, r^{\prime}\right)\right)=h_{j} \rho_{m}\right|\left(A \cap B^{N}\left(0, r^{\prime}\right)\right), \quad j=1, \ldots, m-1
$$

It implies that $L_{0}\left(\rho_{j}, \omega\right)=h_{j}(0) L_{0}\left(\rho_{m}, \omega\right)$ for every $\omega \in T=T_{0} A \cap H_{0}\left(\Gamma_{m}\right)$. Set $\alpha=\left(h_{1}(0), \ldots, h_{m-1}(0), 1\right)$. Then (see $\left.(1.5)\right)$ we have $\operatorname{Levi}_{0}^{M}(T) \subset \alpha \mathbf{R}$. This means the contradiction with (3.2) because $\operatorname{dim} T=q-1$. Therefore for every $r>0$ the intersection $A \cap M \cap B^{N}(0, r)$ is the proper closed subset of $A \cap \Gamma_{m} \cap B^{N}(0, r)$. It implies by the obvious way that $(A \backslash M) \cap B^{N}(0, r)$ is linearly connected for every sufficiently small $r>0$.

Lemma 3.5. Let $M$ be a generic $C^{2}$-manifold of codimension $m \geq 2$ in $\mathrm{C}^{N}$ and $A$ be a complex $q$-dimensional manifold in some neighborhood $U$ of a point $p \in A \cap M$. Suppose that the set $Q_{p}(N-m-q+2)$ is non-empty and (3.2) is satisfied for every $a \in A \cap M \cap U$. Then for every sufficiently small $r>0$, the set $(A \backslash M) \cap B^{N}(p, r)$ is linearly connected and $A \cap M \cap B^{N}(p, r)$ is the nowhere dense subset of $A \cap B^{N}(p, r)$. 
Proof. Without loss of generality we may assume that $p=0$. By Lemma 3.4 it is sufficient to consider the case when $T_{0} A \subset H_{0}(M)$. Let $z^{\prime}=\left(z_{1}, \ldots, z_{q}\right)$ and $\mathbf{C}^{q}\left(z^{\prime}\right) \subset \mathbf{C}^{N}$ is the coordinate plane of variables $z_{1}, \ldots, z_{q}$. After the local biholomorphic change of coordinates we have $A \cap B^{N}(0, r)=\mathbf{C}^{q}\left(z^{\prime}\right) \cap B^{N}(0, r)$. Since $Q_{0}(N-m-q+2)$ is non-empty there exists $\alpha \in S^{m-1}$ such that the restriction of the form $L_{0}(\langle\alpha, \hat{\rho}\rangle, z)$ on $\mathbf{C}^{q}\left(z^{\prime}\right)$ has at least two positive eigenvalues. Let us consider the restriction $\rho_{j}^{\prime}\left(z^{\prime}\right)=\rho_{j} \mid \mathbf{C}^{q}\left(z^{\prime}\right)$. Then

$$
\rho_{j}^{\prime}\left(z^{\prime}\right)=K_{j}\left(z^{\prime}\right)+\overline{K_{j}\left(z^{\prime}\right)}+L_{0}\left(\rho_{j}^{\prime}, z^{\prime}\right)+o\left(\left|z^{\prime}\right|^{2}\right)
$$

where $K_{j}\left(z^{\prime}\right)=\sum u_{\nu \mu}^{j} z_{\nu} z_{\mu}$ and

$$
\mathbf{C}^{q}\left(z^{\prime}\right) \cap M \cap B^{N}(0, r)=\left\{\left|z^{\prime}\right|<r: \rho_{j}^{\prime}\left(z^{\prime}\right)=0, j=1, \ldots, m\right\}
$$

We also may assume that the form $L_{0}\left(\rho_{1}^{\prime}, z^{\prime}\right)$ has no less than two positive eigenvalues. Therefore, after the $\mathbf{C}$-linear change of coordinates in $\mathbf{C}^{q}\left(z^{\prime}\right)$, we have

$$
L_{0}\left(\rho_{1}^{\prime}, z^{\prime}\right)=\left|z_{1}\right|^{2}+\left|z_{2}\right|^{2}+\sum_{\mu=3}^{q} \lambda_{\mu}\left|z_{\mu}\right|^{2}
$$

Let us consider the set $W_{r}=\left\{\left|z^{\prime}\right|<r:\left(\partial \rho_{1}^{\prime} / \partial z_{\nu}\right)\left(z^{\prime}\right)=0, \nu=1,2\right\}$. Then by (3.6), (3.7) we obtain that $W_{r}$ is the real submanifold of codimension $\geq 2$ in $\mathbf{C}^{q}\left(z^{\prime}\right) \cap B^{N}(0, r)$ for every $r>0$. Particularly $W_{r}$ is nowhere dense in $\mathbf{C}^{q}\left(z^{\prime}\right) \cap B^{N}(0, r)$ and $\left(\mathbf{C}^{q}\left(z^{\prime}\right) \backslash W_{r}\right) \cap B^{N}(0, r)$ is linearly connected.

Consider the points $a_{1}$ and $a_{2}$ of $\left(\mathbf{C}^{q}\left(z^{\prime}\right) \backslash M\right) \cap B^{N}(0, r)$. Also choose $r_{j}>$ $0, j=1,2$ such that $B^{N}\left(a_{j}, r_{j}\right) \cap M$ is empty and $B^{N}\left(a_{j}, r_{j}\right) \subset B^{N}(0, r)$. For every $j=1,2$ there exists a point $a_{j}^{\prime} \in \mathbf{C}^{q}\left(z^{\prime}\right) \cap B^{N}\left(a_{j}, r_{j}\right) \backslash\left(M \cup W_{r}\right)$. The points $a_{1}^{\prime}$ and $a_{2}^{\prime}$ can be connected by the (continuous) path $\gamma \subset \mathbf{C}^{q}\left(z^{\prime}\right) \cap B^{N}(0, r) \backslash W_{r}$. In general, $\gamma$ can intersect $M$, but at every point of the intersection all conditions of Lemma 3.4 are satisfied by the definition of $W_{r}$. Then in every neighborhood of $\gamma$ there exists some path $\gamma^{\prime}$ which connects $a_{1}^{\prime}$ and $a_{2}^{\prime}$ and does not intersect $M$. Let $\gamma_{j} \subset B^{N}\left(a_{j}, r_{j}\right)$ be a path which connects $a_{j}$ and $a_{j}^{\prime}, j=1,2$ and $\gamma_{j}$ does not intersect $M$. Then the path $\gamma_{1} \cup \gamma^{\prime} \cup \gamma_{2}$ lies in $\left(\mathbf{C}^{q}\left(z^{\prime}\right) \backslash M\right) \cap B^{N}(0, r)$ and connects $a_{1}$ with $a_{2}$. Therefore the set $(A \backslash M) \cap B^{N}(0, r)$ is linearly connected for every $r>0$ and it is clear from our arguments that $A \cap M \cap B^{N}(0, r)$ is nowhere dense in $A \cap B^{N}(0, r)$. 
Proof of Theorem 3.1. By Theorem 2.13, $A$ analytically continues to the neighborhood $U$ of every point $p \in \bar{A} \cap M$ as a purely $q$-dimensional subset $\tilde{A} \subset U$. Let $b \in(\operatorname{reg} \tilde{A}) \cap M$ where $\operatorname{reg} \tilde{A}$ is the set of regular points of $\tilde{A}$ and $V$ is a neighborhood of $b$ in which $\tilde{A}$ is a complex $q$-dimensional manifold. By Lemma 3.5 we may assume (probably after some shrinking of $V$ ) that $(\tilde{A} \backslash M) \cap V$ is connected. Suppose also that $b \in \bar{A}$. Then, by the definition of the analytic continuation we have $A \cap V \subset(\tilde{A} \backslash M) \cap V$. But since $(\tilde{A} \backslash M) \cap V$ is connected and $\operatorname{dim} A=\operatorname{dim} \tilde{A}$, we obtain by the uniqueness theorem for analytic sets ([Ch2], Section 5.3 , p. 48) that $A \cap V=(\tilde{A} \backslash M) \cap V$. Then by Lemma 3.5 we have $\overline{(\tilde{A} \backslash M) \cap V}=\tilde{A} \cap V$ and consequently $\bar{A} \cap V=\tilde{A} \cap V$. Therefore $\bar{A}$ is an analytical subset in the neighborhood of every point $b \in \bar{A} \cap(\operatorname{reg} \tilde{A}) \cap M$. Then $\bar{A}$ is an analytic purely $q$-dimensional subset of $U \backslash(\operatorname{sng} \tilde{A} \cap M)$ where $\operatorname{sng} \tilde{A}=\tilde{A} \backslash \operatorname{reg} \tilde{A}$ is the set of non-regular points of $\tilde{A}$. But $\operatorname{sng} \tilde{A}$ is a complex analytic subset of $U$ of dimension $<q([\mathrm{Gu}, \mathrm{Ro}]$, Ch. IV, D, cons 4),[Ch2]. Consequently by the Remmert-Stein theorem on the removal singularities [Re, St], [Gu, Ro], [Ch2] $\bar{A}$ is a complex analytic subset of $U$.

As a consequence we obtain our second main result.

Theorem 3.8. Let $M$ be a generic $C^{2}{ }_{-}$manifold of codimension $m \geq 2$ in a domain $\Omega \subset \mathbf{C}^{n}$ and let $A$ be a complex purely $q$-dimensional subset of $\Omega \backslash M$. Suppose that

$$
q-1>\operatorname{def}_{p}(M)
$$

for every $p \in \bar{A} \cap M$. Then the closure $\bar{A}$ of $A$ is an analytic subset of $\Omega$.

Proof. Since $\ell d_{p}^{1}(M) \leq \operatorname{def}_{p}(M)$, then (3.2) is satisfied. Theorem 2.14 and (3.9) imply that $M$ is $(N-m-q+2)$-pseudoconcave at every point $p \in \bar{A} \cap M$. Therefore Theorem 3.8 is a consequence of Theorem 3.1.

Note that usually it is considerably easier to verify (3.9) than conditions of Theorem 3.1. Therefore Theorem 3.8 can be useful for applications.

Remark 3.10. J. Becker [Be] and B. Shiffman [Sh2] proved that $\mathbf{R}^{N}$ and the skeleton of polydisk are removable for $q$-dimensional analytic sets with $q \geq 2$. More generally, E. Chirka [Ch1] showed that if $q-1>\mathrm{CR} \operatorname{dim} M$, then $M$ is removable (some partial case was considered in $[\mathrm{Fu}]$ ). The strongest result was obtained in $[\mathrm{Pi}, \mathrm{Su}]$, where $\ell d_{p}^{m-1}(M)$ was used instead of $\operatorname{def}_{p}(M)$ in (3.9). It is obvious that all these results are consequences of Theorem 3.8. Note also that Theorem 3.8 essentially strengthens these results. To see this, let us consider the manifold $M \subset \mathbf{C}^{2 n}$ from Example 1.9. According to [Pi, Su], $M$ is removable for analytic sets of dimension $\geq(n+1)$. But Theorem 3.8 shows that in reality $M$ is removable for analytic sets of dimension $\geq 3$. 


\section{REFERENCES}

[Be] J. BECKER, Continuing analytic sets across $\mathbf{R}^{n}$, Math. Ann 195 (1972), 103-106.

[Bo, Po] A. Boggess $\&$ J. Polking, Holomorphic extension of CR functions, Duke Math. J. 49 (1982), 757-784.

[Ch1] E.M. ChIRKA, On the removable singularities of analytic sets, Dokl. AN USSR 248 (1979), 47-50, (Russian).

[Ch2] — Complex analytic sets, (Russian), Nauka, Moscow, 1985.

[Ch3] - Introduction to the geometry of CR-manifolds, Uspekhi matem. nauk. 46,1 (1991), 81-164, (Russian).

[Fu] K. Funahashi, On the extension of analytic sets, Proc. Japan. Acad. 54,1 (1978), 25-26.

[Gu, Ro] R. Gunning $\&$ H. Rossi, Analytic functions of several complex variables, Prentice-Hall, Inc., NJ, 1965.

[Ho, Jo] R. Horn \& C. Johnson C., Matrix Analysis, Cambridge Univ. Press, 1986.

[Pi] S.I. Pinchuк, Analytic sets: the reflection principle, "edge of the wedge theorem", the analytic continuation, Preprint, Ufa (1986).

[Pi, Su] S.I. PINchuk, A.B. SukHov, The continuation of analytic sets across real manifolds, Matem. zametki 41,3 (1987), 320-324, (Russian).

[R] R. Rockafellar, Convex Analysis, Princeton Univ. Press, 1970.

[Re, St] R. REMMERT $\&$ K. STEIN, Uber die wesentlichen singularitaten analytischer Mengen, Math. Ann. 126 (1953), 263-306.

[Rot] W. Rothstein, Zur Theorie der analytischen Mannigfaltigkeiten im Raume von n komplexen Veranderlichen, Math. Ann. . 129 (1955), 96-138; (1957), vol. 133, 271-280; (1957), vol. 133, 400-409.

[Sha] B.V. ShaвAт, Introduction to the Complex Analysis, vol. 2 (Russian), Moscow, 1985 .

[Sh1] B. Shiffman, On the removal of singularities of analytic sets, Michigan Math. J. 15 (1968), 111-120.

[Sh2] - On the continuation of analytic sets, Math. Ann. 185 (1970), 1-12.

Department of Mathematics

Bashkirian University

Frunze str. 32

450074, Ufa, Russia

Received: January 22nd, 1992. 